\title{
Small bowel adenocarcinoma arising in a patient with hereditary hemorrhagic telangiectasia: A case report
}

\author{
YUICHIRO YOSHIOKA, HIROAKI NOZAWA, JUNICHIRO TANAKA, TAKESHI NISHIKAWA, \\ TOSHIAKI TANAKA, TOMOMICHI KIYOMATSU, KAZUSHIGE KAWAI, KEISUKE HATA, SHINSUKE KAZAMA, \\ HIRONORI YAMAGUCHI, SOICHIRO ISHIHARA, EIJI SUNAMI, JOJI KITAYAMA and TOSHIAKI WATANABE
}

Department of Surgical Oncology, University of Tokyo, Tokyo 113-8655, Japan

Received November 23, 2014; Accepted October 21, 2015

DOI: $10.3892 / 01.2016 .4173$

\begin{abstract}
Patients with hereditary hemorrhagic telangiectasia (HHT) are reportedly at a lower overall risk of malignancies, and small bowel adenocarcinoma (SBA) arising in a HHT patient is extremely rare. In this study, the case of a 37-year-old female with HHT who developed a poorly differentiated jejunal adenocarcinoma five years after ileocecal resection for multiple colonic adenomas is presented. The patient underwent curative resection of the cancer invading the ileum and the mesentery of the transverse colon, but had to overcome critical complications perioperatively, stemming from HHT-associated peripheral capillary dilatation and arteriovenous malformation, including nosebleeds and possible infusion-induced air embolism through pulmonary shunts. The patient subsequently received adjuvant chemotherapy including capecitabine and oxaliplatin for 6 months, and currently remains alive without any evidence of recurrence 12 months after the second surgery. This patient with SBA was an instructive case demonstrating the necessity of careful attention during major surgery in HHT.
\end{abstract}

\section{Introduction}

Hereditary hemorrhagic telangiectasia (HHT), also known as Osler-Weber-Rendu disease, is an autosomal dominant disorder characterized by cutaneous and/or mucosal telangiectasias and arteriovenous malformations (AVMs), with a prevalence rate of 1-2 patients per 10,000 individuals worldwide (1). HHT is clinically diagnosed using the Curaçao criteria, which was

Correspondence to: Dr Hiroaki Nozawa, Department of Surgical Oncology, University of Tokyo, 7-3-1 Hongo, Bunkyo-ku, Tokyo 113-8655, Japan

E-mail: hiroanozawa-gi@umin.ac.jp

Abbreviations: HHT, hereditary hemorrhagic telangiectasia; CT, computed tomography; SBA, small bowel adenocarcinoma; FAP, familial adenomatous polyposis

Key words: chemotherapy, hereditary hemorrhagic telangiectasia, small bowel adenocarcinoma, surgical management, complications established in 1999 (2). Approximately 80\% of HHT patients exhibit a mutation in endoglin or activin A receptor-like kinase 1 genes. Repeated nosebleeds due to the mucosal telangiectasias are the most common somatic symptom and present a major HHT-related health concern that affects patient quality of life (3). At present, an optimal treatment strategy for HHT has not yet been established. However, in patients that exhibit severe bleeding, infection and hypoxia, surgery is usually required $(3,4)$. The prognosis of HHT patients depends on the presence of the infection associated with AVMs, including organ abscesses and sepsis (1). Notably, the mean lifespan of HHT patients has been found to be shortened when compared with controls (63.2 vs. 70.0 years) (4). To date, only a few cases of tumors arising in the small bowel have been reported $(5,6)$. In this study, the first case of a patient with HHT to develop cancer of the jejunum following surgical removal of multiple colonic adenomas is presented.

\section{Case report}

A 32-year-old female underwent a laparoscopy-assisted ileocecal resection for multiple polyps in the cecum and appendix veriforme at the University of Tokyo Hospital, (Tokyo, Japan), as reported in a previous study (7). The patient was subsequently diagnosed with HHT. Five years later, she visited a local practitioner complaining of appetite loss which had lasted for two months, recent abdominal pain, and intermittent vomiting. Shortly thereafter, the patient was referred back to the University of Tokyo Hospital to receive treatment for obstructive ileus, which was managed by intravenous hydration without oral intake. A decompression tube was not inserted through the nose in order to avoid nosebleeds.

Laboratory examinations performed on admission revealed normal findings, with the exception of anemia (hemoglobin, $8.3 \mathrm{~g} / \mathrm{dl}$ ) and malnutrition (serum protein, $4.7 \mathrm{~g} / \mathrm{dl}$; albumin, $2.3 \mathrm{~g} / \mathrm{dl}$ ). Progressive anemia due to frequent nosebleeds and melena originating from the tumor necessitated a blood transfusion prior to surgery. An abdominal computed tomography (CT) scan revealed a small bowel tumor, $\sim 10 \mathrm{~cm}$ in diameter, directly behind the transverse mesocolon (Figs. 1 and 2). Magnetic resonance imaging revealed a tumor whose border was highly enhanced by contrast and which demonstrated high intensity 


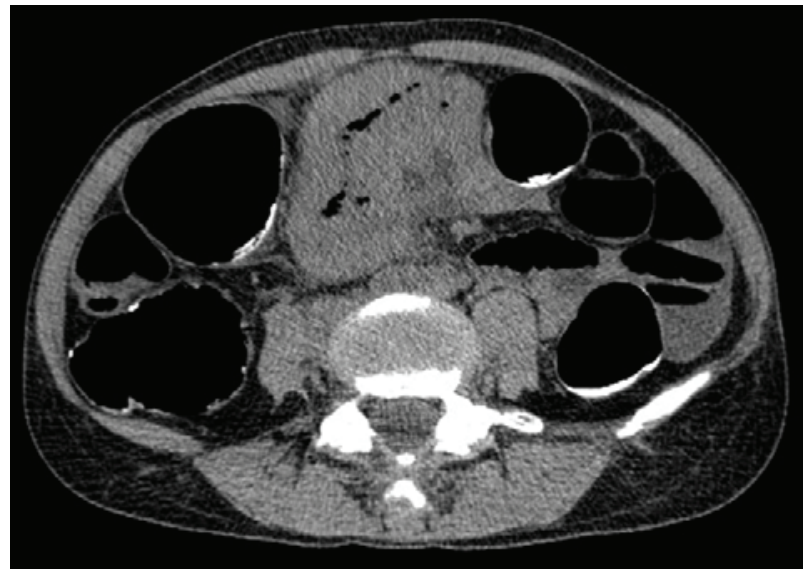

Figure 1. Axial view of abdominal computed tomography scan.

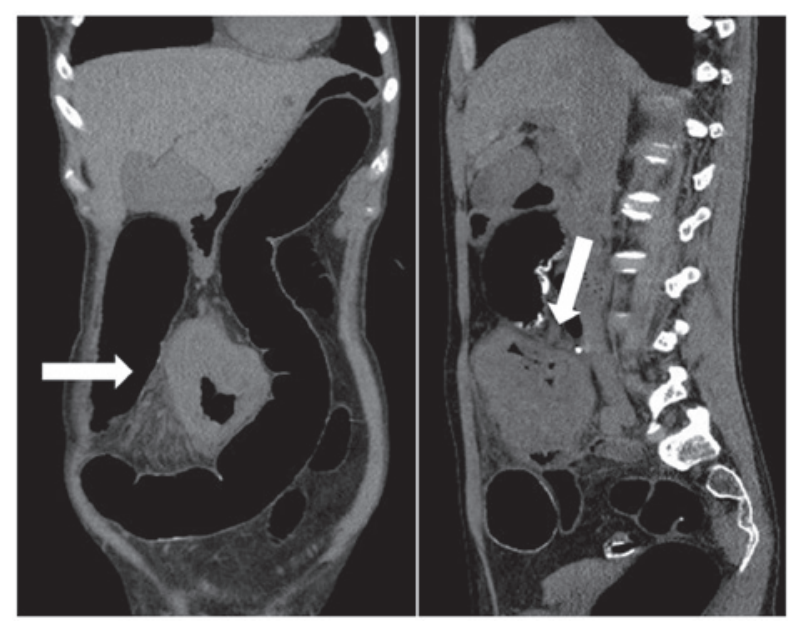

Figure 2. Coronal (left) and sagittal views (right) of computed tomography scans. The arrow indicates the small bowel tumor.

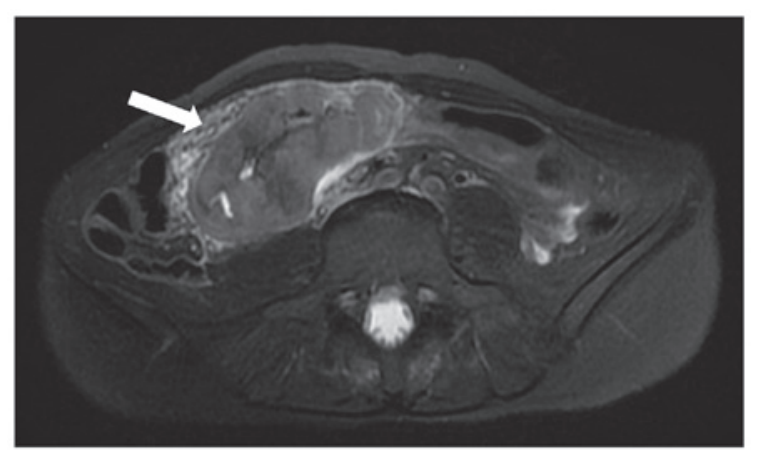

Figure 3. Magnetic resonance imaging. The arrow indicates the small bowel tumor.

in diffusion-weighted imaging (Fig. 3). Positron emission CT revealed a maximum standardized uptake value of 14.9 in the lesion, suggestive of a malignant tumor. No distant organ metastasis was demonstrated (image not shown).

The patient experienced loss of consciousness twice when the infusion bag for hyper-alimentation was changed, which was accessed from the right internal jugular vein. Image studies of the patient's head did not reveal significant

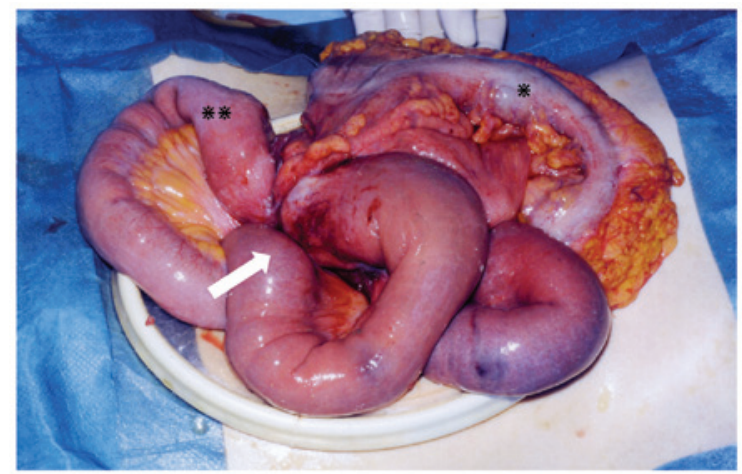

Figure 4. Intraoperative findings. The small bowel tumor is indicated by the arrow. "Transverse colon, ${ }^{* *}$ ileum.

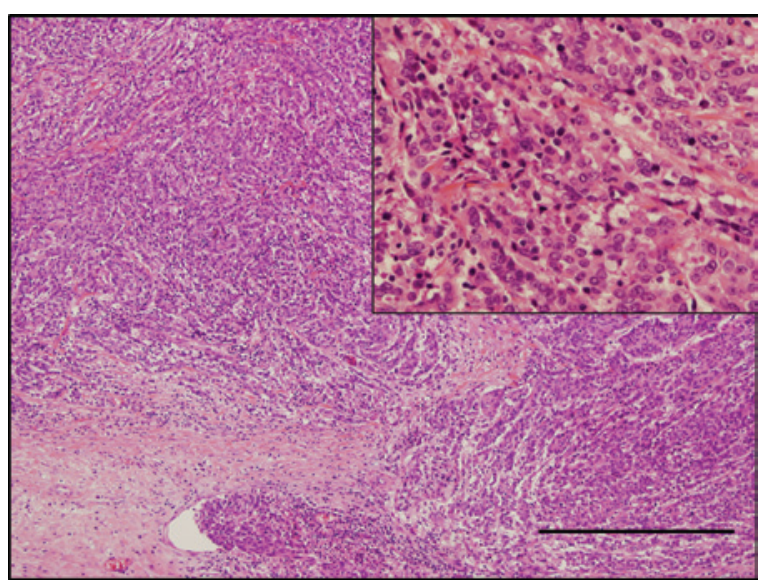

Figure 5. Histological examination of the resected specimen. Hematoxylin and eosin staining. The bar indicates $500 \mathrm{~mm}$. Magnification: Main image, $x 20$; inset image, $x 400$.

ischemic changes or metastatic lesions. Considering the presence of arteriovenous fistulas in the patient's lungs (7), it was notable that micro air bubbles from the infusion line had passed through the shunts to the cerebral arteries, causing transient ischemic attack. The patient was free from ischemic episodes after stopping central vein hyper-alimentation.

Resection of the tumor-bearing segment of the jejunum (30 $\mathrm{cm}$ from the Treitz ligament) as well as right hemicolectomy was performed since the tumor had invaded the terminal ileum as well as the mesenterium of the transverse colon (Fig. 4). The resected specimen was histologically diagnosed as poorly differentiated adenocarcinoma (Fig. 5) with prominent lymphatic and venous infiltration. One of the twelve regional lymph nodes dissected was affected by cancer.

In addition to epistaxis, the patient experienced wound infection postoperatively, which could be treated conservatively. Her postoperative recovery was otherwise uneventful. She received an intravenous infusion of oxaliplatin $\left(123 \mathrm{mg} / \mathrm{m}^{2}\right)$ on day 1 plus oral capecitabine $\left(920 \mathrm{mg} / \mathrm{m}^{2}\right)(\mathrm{CapeOx})$ twice daily for 2 weeks in a 3-week cycle as adjuvant chemotherapy for 6 months (a total of 8 cycles); chemotherapy-related peripheral neurotoxicity and neutropenia could be managed. Due to severe neuropathy the dosage of oxaliplatin was reduced by $20 \%$ for the final two cycles. The patient is currently alive without evidence of recurrence 12 months after the surgery. 
Written informed consent was obtained from the patient for publication of the present study.

\section{Discussion}

Small bowel adenocarcinomas (SBAs) account for $<5 \%$ of gastrointestinal cancers worldwide (8). Genetic predisposition to SBAs has been indicated; patients with familial adenomatous polyposis, Lynch syndrome and Peutz-Jeghers syndrome have a relatively higher risk of SBA than the general population, suggesting genetic alterations predisposed to cancer development (9).

Genetic analyses have revealed that abnormalities in endoglin family genes cause HHT (10). In an in vivo experiment, the overexpression of endoglins in prostate cancer cells revealed growth-inhibiting effects on tumor cells (11-14). However, other animal studies have revealed that protein promotes angiogenesis in a tumor microenvironment, causing accelerated metastasis (15). Therefore, it is an interesting question of whether HHT manifests cancer-prone phenotypes or not. A clinical observational study demonstrated that reduced endoglin expression in HHT is associated with a lower frequency of common malignancies, including lung, prostate and colorectal cancer, and a more favorable cancer-specific survival (10). Based on these findings, the current patient's case is rare. To the best of our knowledge, this is the first HHT patient to develop cancer of the small intestine, excluding the duodenum (5).

The current case illustrates several significant points in the perioperative care of patients with HHT. First, in the surgical treatment of a gastrointestinal malignancy, frequent nosebleeds may hinder the appropriate management of a possible perioperative ileus since the insertion of a nasogastric or long tube may exacerbate anemia. In addition, the existence of an arteriovenous shunt easily causes an arterial embolism during intravenous infusion, particularly when using the central vein. In the current case, significant infarction was avoided by switching to peripheral vein nutrition. Therefore, special management is required for HHT patients who undergo gastrointestinal surgery.

In general, SBA exhibits a poor prognosis (9). In addition, poorly differentiated adenocarcinoma, as in the current case, is one of the predictors of poor outcome (8). There have been only a limited number of studies on the subject of adjuvant chemotherapy for SBAs, most of which are retrospective $(16,17)$. Similarly, the majority of published studies pertaining to palliative chemotherapy for advanced SBAs are retrospective and limited in number. The best outcome was a median overall survival of 20 months achieved in a phase II trial of CapeOx in patients with advanced SBAs (18). Adjuvant CapeOx treatment was selected in the current case based on the results of the above-mentioned study as well as by extrapolating from relapse-preventing effects of CapeOx for colon cancer (19). Furthermore, CapeOx is advantageous in terms of minimizing the infusion time of chemotherapeutic drugs, and appears to be an ideal regimen for cancer patients with HHT.

In conclusion, the present study reported the case of an HHT patient diagnosed with jejunum cancer, an extremely uncommon condition according to epidemiological studies. This study shows that HHT is a disorder that may cause clinically significant complications due to peripheral artery dilatation and arteriovenous malformation, thereby suggesting the necessity of cautious management during perioperative care.

\section{References}

1. Guttmacher AE, Marchuk DA and White RI Jr: Hereditary hemorrhagic telangiectasia. N Engl J Med 333: 918-924, 1995.

2. Shovlin CL, Guttmacher AE, Buscarini E, Faughnan ME, Hyland RH, Westermann CJ, Kjeldsen AD and Plauchu H: Diagnostic criteria for hereditary hemorrhagic telangiectasia (Rendu-OslerWeber syndrome). Am J Med Genet 91: 66-67, 2000.

3. Arthur H, Geisthoff U, Gossage JR, Hughes CC, Lacombe P, Meek ME, Oh P, Roman BL, Trerotola SO, Velthuis S and Wooderchak-Donahue W: Executive summary of the 11th HHT international scientific conference. Angiogenesis 18: 511-524, 2015.

4. Sabbà C, Pasculli G, Suppressa P, D'Ovidio F, Lenato GM, Resta F, Assennato G and Guanti G: Life expectancy in patients with hereditary haemorrhagic telangiectasia. QJM 99: 327-334, 2006.

5. Korzenik J, Chung DC, Digumarthy S and Badizadegan K: Case records of the Massachusetts General Hospital. Case 33-2005. A 43-year-old man with lower gastrointestinal bleeding. N Engl J Med 353: 1836-1844, 2005.

6. Kato S and Ichihara K: Hereditary hemorrhagic telangiectasia with malignant lymphoma. An autopsy case. Acta Pathol Jpn 38: 383-391, 1988.

7. Kaneko M, Nozawa H, Kitayama J, Sunami E, Akahane M, Yamauchi N, Furukawa Y and Nagawa H: A case of hereditary hemorrhagic telangiectasia (Osler-Weber-Rendu disease) with multiple polyps arising in the cecum and appendix. Acta Gastroenterol Belg 74: 352-354, 2011.

8. Neugut AI, Jacobson JS, Suh S, Mukherjee R and Arber N: The epidemiology of cancer of the small bowel. Cancer Epidemiol Biomarkers Prev 7: 243-251, 1998.

9. Aparicio T, Zaanan A, Svrcek M, Laurent-Puig P, Carrere N, Manfredi S, Locher C and Afchain P: Small bowel adenocarcinoma: epidemiology, risk factors, diagnosis and treatment. Dig Liver Dis 46: 97-104, 2014.

10. Duarte CW, Murray K, Lucas FL, Fairfield K, Miller H, Brooks P and Vary CP: Improved survival outcomes in cancer patients with hereditary hemorrhagic telangiectasia. Cancer Epidemiol Biomarkers Prev 23: 117-125, 2014.

11. Liu Y, Jovanovic B, Pins M, Lee C and Bergan RC: Over expression of endoglin in human prostate cancer suppresses cell detachment, migration and invasion. Oncogene 21: 8272-8281, 2002.

12. Lakshman M, Huang X, Ananthanarayanan V, Jovanovic B, Liu Y, Craft CS, Romero D, Vary CP and Bergan RC: Endoglin suppresses human prostate cancer metastasis. Clin Exp Metastasis 28: 39-53, 2011.

13. Craft CS, Romero D, Vary CP and Bergan RC: Endoglin inhibits prostate cancer motility via activation of the ALK2-Smad1 pathway. Oncogene 26: 7240-7250, 2007.

14. Romero D, Terzic A, Conley BA, Craft CS, Jovanovic B, Bergan RC and Vary CP: Endoglin phosphorylation by ALK2 contributes to the regulation of prostate cancer cell migration. Carcinogenesis 31: 359-366, 2010.

15. Romero D, O’Neill C, Terzic A, Contois L, Young K, Comley BA, Bergan RC, Brooks PC and Vary CP: Endoglin regulates cancer-stromal cell interactions in prostate tumors. Cancer Res 71: 3482-3493, 2011.

16. Koo DH, Yun SC, Hong YS, Ryu MH, Lee JL, Chang HM, Kang YK, Kim SC, Han DJ, Lee YJ and Kim TW: Adjuvant chemotherapy for small bowel adenocarcinoma after curative surgery. Oncology 80: 208-213, 2011.

17. Overman MJ, Lopetz S, Lin E, Abbruzzese JL and Wolff RA: Is there a role for adjuvant therapy in resected adenocarcinoma of the small intestine. Acta Oncol 49: 474-479, 2010.

18. Overman MJ, Varadhachary GR, Kopetz S, Adinin R, Lin E, Morris JS, Eng C, Abbruzzese JL and Wolff RA: Phase II study of capecitabine and oxaliplatin for advanced adenocarcinoma of the small bowel and ampulla of vater. J Clin Oncol 27: 2598-2603, 2009.

19. Haller DG, Tabernero J, Maroun J, de Braud F, Price T, Van Cutsem E, Hill M, Gilberg F, Rittweger K and Schmoll HJ: Capecitabine plus oxaliplatin compared with fluorouracil and folinic acid as adjuvant therapy for stage III colon cancer. J Clin Oncol 29: 1465-1471, 2011. 